\title{
Clavien-Dindo Grade II
}

National Cancer Institute

\section{Source}

National Cancer Institute. Clavien-Dindo Grade II. NCI Thesaurus. Code C121448.

A complication requiring pharmacological treatment with drug other than such allowed for Grade I complications. 